\title{
Mechanism for reversed photoemission core-level shifts of oxidized Ag
}

\author{
Henrik Grönbeck, ${ }^{1, *}$ Simon Klacar, ${ }^{1}$ Natalia M. Martin, ${ }^{2}$ Anders Hellman, ${ }^{1}$ Edvin Lundgren, ${ }^{2}$ and Jesper N. Andersen ${ }^{2}$ \\ ${ }^{1}$ Department of Applied Physics and Competence Centre for Catalysis, Chalmers University of Technology, SE-412 96, Göteborg, Sweden \\ ${ }^{2}$ Division of Synchrotron Radiation Research, Lund University, Box 118, SE-221 00, Sweden
}

(Received 22 August 2011; revised manuscript received 15 March 2012; published 29 March 2012)

\begin{abstract}
Density functional theory calculations and high-resolution core-level spectroscopy are used to explore the remarkable observation of decreased $\mathrm{Ag} 3 d$ binding energy upon silver oxidation. The shift in $\mathrm{Ag} 3 d$ binding energy is investigated at different degrees of oxidation and compared to results for $\mathrm{Pd} 3 d$, which exhibits a normal shift. Analysis of initial-state effects and valence electronic structure shows that the onsite $\mathrm{Ag}$ core potential is insensitive to oxidation despite a clear metal-to-oxygen charge transfer. The substantial negative shift for oxidized $\mathrm{Ag}$ is instead attributed to final-state effects as screening of the core-hole occurs in metal $s$ states of bonding character.
\end{abstract}

DOI: 10.1103/PhysRevB.85.115445

PACS number(s): 82.80.Pv, 73.20.At

\section{INTRODUCTION}

The response of core electrons to rehybridization and charge transfer in the valence region make shifts in binding energies valuable signatures of chemical bonds. Photoelectron emission spectroscopy has, thanks to the sensitivity of the local atomic environment, developed into a standard technique for chemical analysis. ${ }^{1}$ High-resolution core-level spectroscopy (HRCLS) is, furthermore, frequently used in combination with diffraction techniques and scanning probe microscopy for structural determinations. $^{2-4}$ However, as similar core-level binding energies, in principle, could have different origins, it has proven essential to compare experimental results with predictions of models for core-level shifts ${ }^{5-7}$ (CLS) or explicit first-principles calculations.

One long-standing puzzle in the analysis of CLS of late transition metals is the oxidation-induced shift of $\mathrm{Ag} 3 d$. In contrast to other transition metals, the core levels of $\mathrm{Ag}$ are shifted to lower binding energies upon oxidation. ${ }^{4,8-10}$ This observation is counterintuitive in the sense that it contradicts the electrostatic potential model that often has been used to interpret chemical shifts. ${ }^{5}$ In this model, the shift of a core state at site $i$ is given by

$$
\Delta E_{i}=k q_{i}+\sum_{j \neq i} \frac{q_{j}}{R_{i j}} .
$$

Here, $q_{i}$ is the net charge on atom $i, k$ is the Coulomb repulsion integral between core and valence states, ${ }^{6}$ and $R_{i j}$ is the distance between the nuclei $i$ and $j$. A positive (negative) shift is predicted in cases where the net charge on the site of interest is positive (negative). Because of the high electronegativity of oxygen, the model predicts positive CLS of Ag upon oxidation. Although the simplicity of the electrostatic potential model is appealing, its limitations are documented. ${ }^{10,11}$ One illustrative example is CLS of binary alloys ${ }^{12}$ where, for example, negative shifts have been measured ${ }^{13}$ and calculated ${ }^{12}$ for both $\mathrm{Pd}$ and $\mathrm{Ag}$ in random $\mathrm{Pd}-\mathrm{Ag}$ alloys. The change in onsite electrostatic potential is one part of what generally is referred to as initial-state effects. The initial-state effects may also include changes in the Fermi energy, intra-atomic charge transfer, and charge redistribution owing to bond rehybridization. ${ }^{11}$

In addition to initial-state effects, the photoemission experiment measures electronic relaxation around the created core hole. This relaxation $\left(\Delta E_{i}^{r}\right)$ is often referred to as a final-state effect. A more complete representation of the core-level shift $E_{i}^{C L S}$ is, consequently,

$$
E_{i}^{\mathrm{CLS}}=\Delta E_{i}-\Delta E_{i}^{r} .
$$

It is unclear whether the observations of reversed CLS for oxidized silver should be attributed to initial- or final-state effects (or a combination).

Here, we perform HRCLS measurements of oxidized $\operatorname{Ag}(111)$ and investigate the origin of the reversed CLS by density functional theory (DFT) calculations. To clearly illustrate this special case, comparative measurements and calculations are performed for Pd. Palladium and silver are neighbors in the periodic table of elements, and the corresponding electronic configurations for the gas phase atoms are $4 d^{10} s^{0}$ and $4 d^{10} s^{1}$, respectively. Palladium in the condensed phase adopts a configuration close to $4 d^{9} s^{1}$.

\section{COMPUTATIONAL AND EXPERIMENTAL METHODS}

DFT is employed in the implementation with plane waves and pseudopotentials. ${ }^{14,15}$ The spin-polarized Perdew-BurkeErnzerhof (PBE) approximation is used for the exchange and correlation (xc) functional, ${ }^{16}$ and ultrasoft scalar-relativistic pseudopotentials are used to describe the interaction between the valence electrons and the core. ${ }^{17}$ The number of electrons treated variationally for each element are $\operatorname{Pd}(10), \operatorname{Ag}(11)$, and $\mathrm{O}(6)$. A plane-wave kinetic energy cutoff of $28 \mathrm{Ry}$ is used to expand the Kohn-Sham orbitals. With this approach, the lattice constants of Pd and $\mathrm{Ag}$ are calculated to be 3.93 and $4.14 \AA$, respectively.

Adsorption of oxygen on $\mathrm{Pd}(111)$ and $\mathrm{Ag}(111)$ is investigated in a $p(2 \times 2)$ surface cell. The surface is represented by five atomic layers. Repeated slabs are separated by $14 \AA$ of vacuum. Reciprocal space integration over the Brillouin zone is approximated with a finite sampling of 13 special $k$ points. Structural optimization is performed without any constraints, and the structures are regarded as converged when the largest element of the gradient is lower than $0.05 \mathrm{eV} / \AA$. The optimized bond lengths around the metal atoms in the central layer are within $0.01 \%$ of the corresponding bulk value. To facilitate proper comparisons with experimental data, we study also 
the structurally resolved surface oxide of $\operatorname{Pd}(100)$ (Ref. 2) and oxidized $\operatorname{Ag}(111))^{3,4}$ The surface oxide on $\operatorname{Pd}(100)$ has a $\sqrt{5} \times \sqrt{5} R 27^{\circ}$ (hereafter denoted $\sqrt{5}$ ) superstructure. This geometry is modeled by an oxide monolayer supported on a five-layer $\operatorname{Pd}(100)$ slab. The oxidized $\mathrm{Ag}(111)$ surface has a $p(4 \times 4)$ unit cell, which again is represented by five atomic layers in addition to the oxidized first layer. The Brillouin zone is sampled by eight special $k$ points for both oxidized surfaces.

The surface core-level shifts are evaluated by the use of a pseudopotential that is generated with an electron hole in the $3 d$ shell. $^{18}$ This approach assumes complete screening of the core hole. This is justified for the present systems by the favorable comparisons to experiments for the oxidized surfaces. The CLS are calculated with respect to a bulk reference, represented by an atom in the center of the slab.

The HRCLS experiments are carried out at Beamline I311 (Ref. 19) at the Swedish synchrotron radiation facility MAX-Lab in Lund, Sweden. All spectra are collected in normal emission with photon energies of 400 and $430 \mathrm{eV}$ for the $\mathrm{Pd} 3 d_{5 / 2}$ and $\mathrm{Ag} 3 d_{5 / 2}$, respectively, and the binding energies are calibrated to the Fermi edge. The samples are cleaned by repeated cycles of sputtering with $\mathrm{Ar}^{+}$ions and annealing up to 850 and $1150 \mathrm{~K}$ for $\mathrm{Ag}$ and $\mathrm{Pd}$, respectively. The surface cleanliness is checked by low-energy electron diffraction (LEED) and x-ray photoemission spectroscopy (XPS). Atomic oxygen (produced with a commercial thermal gas cracker) is used to oxidize $\operatorname{Ag}(111)$, whereas molecular oxygen is used for $\operatorname{Pd}(100)$. The samples are oxidized until the $d_{5 / 2}$ spectra resemble the spectra previously reported in the literature for $\mathrm{Ag}(111)$ (Ref. 4) and $\operatorname{Pd}(100) .{ }^{20}$

\section{RESULTS AND DISCUSSION}

To exemplify the anomalous CLS for Ag and simultaneously demonstrate the performance of the applied methodology, we discuss first the CLS of the structurally known surface oxide of $\mathrm{Pd}(100)$ and oxidized $\mathrm{Ag}(111)$. The core-level spectra are reported in Fig. 1, where also the structures of the oxidized surfaces are shown. The $\sqrt{5}$ surface oxide of $\operatorname{Pd}(100)$ [Fig. 1(c)] consists of one $\mathrm{PdO}(101)$ layer supported on an unreconstructed $\mathrm{Pd}(100)$. The PdO(101) layer has two types of Pd atoms; $\mathrm{Pd}$ is either coordinated to two or four $\mathrm{O}$ atoms. However, each $\mathrm{Pd}$ atom has a unique position in the surface cell. The $p(4 \times 4)$ structure of oxidized $\mathrm{Ag}(111)$ [Fig. 1(f)] is composed of a stoichiometric $\mathrm{Ag}_{12} \mathrm{O}_{6}$ overlayer supported on an unreconstructed $\operatorname{Ag}(111)$ surface. The unit cell consists of two planar $\mathrm{Ag}_{6}$ triangles. One is placed with the atoms in fcc sites, whereas the atoms of the other occupy hcp sites. The furrow between the triangles provides adsorption sites for $\mathrm{O}$ atoms.

The measured Pd $3 d_{5 / 2}$ CLS for clean Pd(100) [Fig. 1(a)] is deconvoluted with two components; one that corresponds to the bulk of the crystal $(335.1 \mathrm{eV})$ and one surface component shifted $-0.42 \mathrm{eV}$ with respect to the bulk. For the $\sqrt{5}$ structure, three components are visible in addition to the bulk signature; one at $-0.31 \mathrm{eV}$ and two at higher binding energies, namely, +0.38 and $+1.30 \mathrm{eV}$, respectively. These results are in good agreement with previous reports. ${ }^{20,21}$ The calculated Pd $3 d$ CLS for the surface component in clean $\mathrm{Pd}(100)$ is $-0.36 \mathrm{eV}$. For the oxidized surface, the atoms in

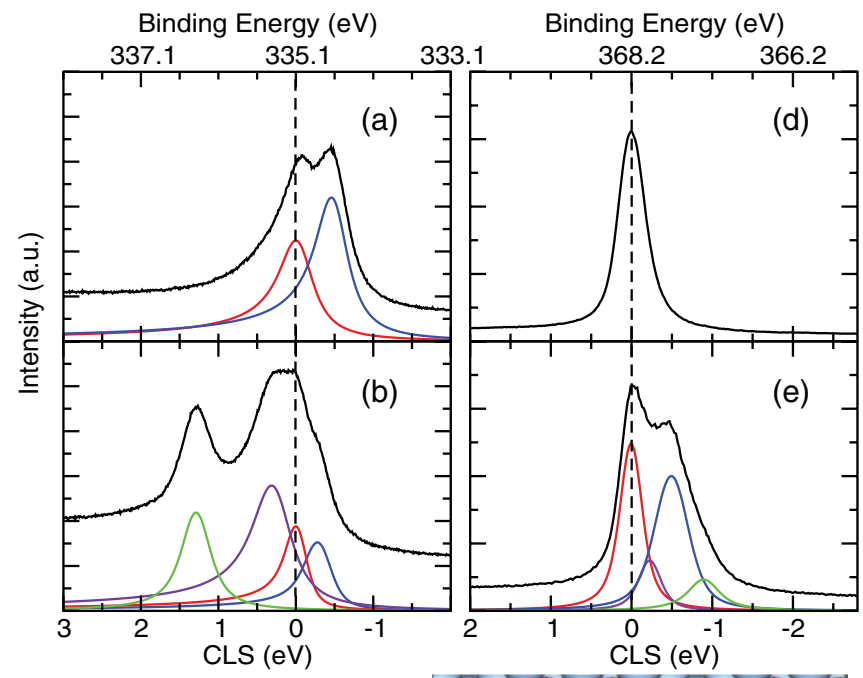

(c)
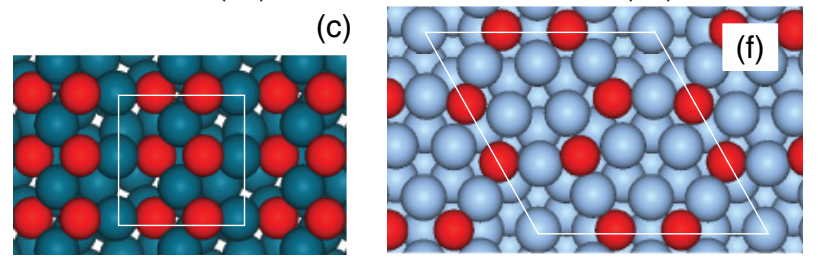

FIG. 1. (Color online) Experimental $3 d_{5 / 2}$ photoemission spectrum of $\operatorname{Pd}(100)(a), \sqrt{5}(b), \operatorname{Ag}(111)(d)$, and $p(4 \times 4)(e)$. The absolute binding energies are reported as well as shifts with respect to the bulk component. Structures of oxidized $\operatorname{Pd}(100)$ and $\operatorname{Ag}(111)$ are shown in (c) and (f), respectively. Atomic color codes: dark blue $(\mathrm{Pd})$, bright blue $(\mathrm{Ag})$, and red $(\mathrm{O})$. The surface cells are indicated by white lines.

the first surface layer of $\operatorname{Pd}(100)$ are calculated to have shifts grouped at -0.2 and $+0.2 \mathrm{eV}$, respectively. ${ }^{22}$ Atoms in the surface oxide that are coordinated to two oxygen atoms have theoretical CLS of +0.34 and $+0.45 \mathrm{eV}$, respectively. The $\mathrm{Pd}$ atoms that coordinate to four oxygen atoms have the largest shifts, namely, +1.17 and $+1.21 \mathrm{eV}$. The results are in good agreement with the experimental results, as well as previous theoretical reports. ${ }^{20}$

For the clean $\operatorname{Ag}(111)$ surface [Fig. 1(d)], the surface and bulk components are close (within $0.1 \mathrm{eV}$ ) and the measurements are unable to resolve the two components. In the oxidized $p(4 \times 4)$ structure, deconvolution yields four features that are assigned to bulk (at a binding energy of $368.2 \mathrm{eV}$ ), and three peaks shifted by $-0.23,-0.60$, and $-0.90 \mathrm{eV}$ with respect to the bulk component. Theoretically, the CLS of the surface atoms in the clean $\mathrm{Ag}(111)$ are calculated to be $-0.07 \mathrm{eV}$. For the $p(4 \times 4)$ oxide, the $\mathrm{Ag} 3 d \mathrm{CLS}$ of the atoms in the two triangles are calculated to be $-0.60 \mathrm{eV}$, whereas the shifts for $\mathrm{Ag}$ atoms in the furrows between the $\mathrm{Ag}_{6}$ clusters are calculated to be $-0.30 \mathrm{eV}$. The remaining atoms in the topmost $\operatorname{Ag}(111)$ layer have CLS of about $-0.1 \mathrm{eV}$. Thus, there is a good match (within $0.1 \mathrm{eV}$ ) between the present calculations and the experiments. The experimental feature at $-0.90 \mathrm{eV}$ is not present in the calculations and is ascribed to small amounts of coexisting structures with higher oxygen content. ${ }^{23}$

The experimental and theoretical results for the two oxidized surfaces illustrate the marked difference of the $3 d$ CLS for Pd and Ag. The shifts for Pd are normal; shifts for 
the metallic surface atoms are negative, whereas oxidized Pd atoms have positive shifts. Moreover, the largest positive shifts are obtained for atoms which could be expected to be in the highest oxidation state (atoms that are coordinated to four $\mathrm{O}$ atoms). The situation for $\mathrm{Ag}$ is reversed. The $3 d$ binding energies of atoms in the $\mathrm{Ag}_{12} \mathrm{O}_{6}$ overlayer are lower than those of the first unreconstructed $\operatorname{Ag}(111)$ surface layer. To explore this peculiar feature in detail and simultaneously make clear connections between $\mathrm{Pd}$ and $\mathrm{Ag}$, we have investigated oxygen adsorption on unreconstructed $\mathrm{Pd}(111)$ and $\mathrm{Ag}(111)$.

$\mathrm{O}$ adsorption is investigated in a $p(2 \times 2)$ surface cell with an $\mathrm{O}$ coverage of $0.25,0.5$, and 0.75 . The $\mathrm{O}$ atoms are placed in fcc sites so as to alter the number of oxygen bonds for one of the atoms in the surface cell. As the focus of this study is to understand the origin of the CLS, only one adsorption configuration was considered for each coverage. The studied adsorption configurations are shown in Figs. 2(a)-2(c).

The calculated CLS are reported in Fig. 2(d). For clean $\operatorname{Pd}(111)$ and $\operatorname{Ag}(111)$ surfaces, we obtain negative shifts of -0.28 and $-0.07 \mathrm{eV}$, respectively. Negative shifts for surface atoms of late transition metal atoms have in the past been rationalized by the electronic configuration of the final state..$^{24,25}$ The screening configurations of Pd and Ag are $d^{10} s^{1}$ and $d^{10} s^{2}$, respectively. The screening in both Pd and Ag takes place in antibonding states, which makes the creation of a core

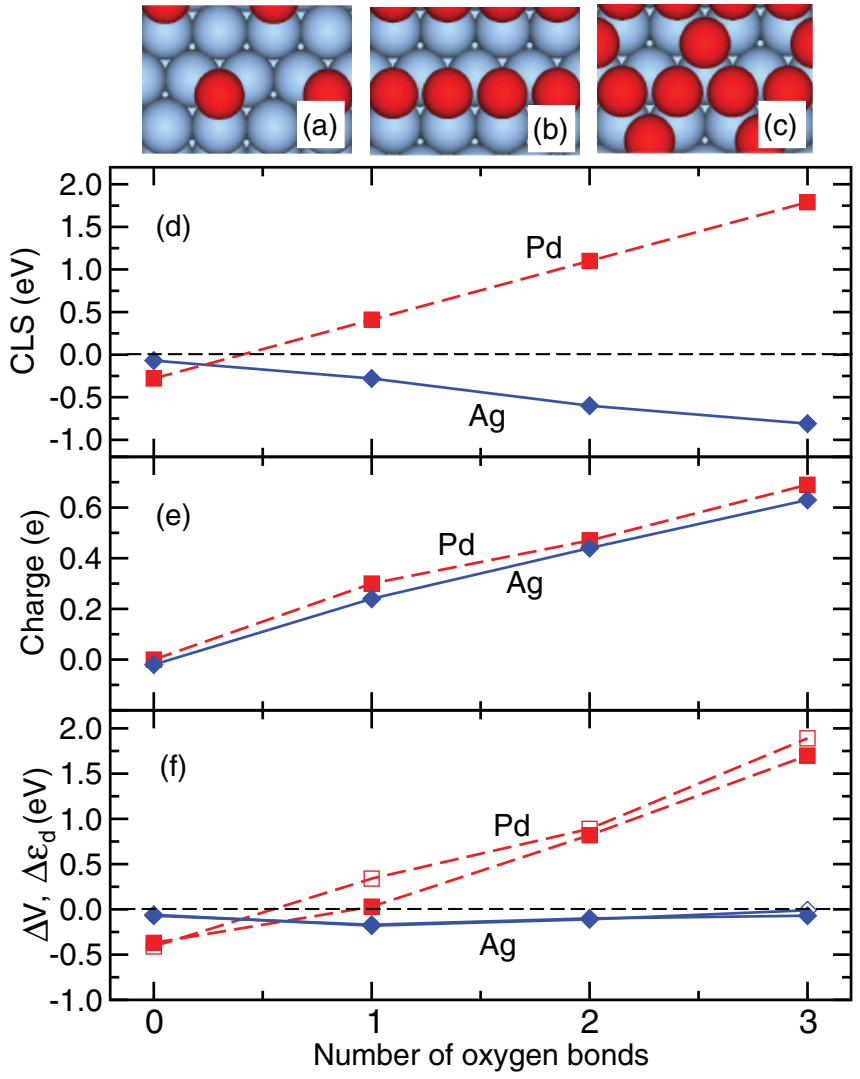

FIG. 2. (Color online) Structural models for $\mathrm{O}$ adsorption at 0.25 (a), 0.5 (b), and 0.75 (c) coverage. CLS (d), charge (e), and $\Delta \varepsilon_{d}$ and $\Delta V$ (see text) (f) for $\mathrm{Pd}$ and $\mathrm{Ag}$ as a function of oxygen-metal bonds. The results for $\mathrm{Pd}(\mathrm{Ag})$ are reported with squares (diamonds). Filled symbols in (f) represent $\Delta \varepsilon_{d}$, whereas open symbols are calculations of $\Delta V$. hole in the surface favorable (lower total energy) as compared to a core hole in a bulk environment. The larger negative shift of Pd is understood from the fact that screening in $d$ states is more efficient than screening in $s$-derived states. ${ }^{24,25}$

The trends for the two metals upon oxidation are opposite. For Pd, the CLS for the atom with one, two, and three oxygen bonds are calculated to be $0.41,1.10$, and $1.79 \mathrm{eV}$, respectively. There is a linear relation between the number of oxygen bonds and the CLS. Also, for Ag, a linear dependence is found, however, the sign of the relation is reversed; the most negative CLS is calculated for the most oxidized Ag atom. The CLS for the atom with one, two, and three oxygen bonds are calculated to be $-0.28,-0.60$, and $-0.81 \mathrm{eV}$, respectively. That a higher coordination of $\mathrm{O}$ atoms yields a more oxidized metal atom is confirmed by a Bader charge analysis ${ }^{26,27}$ reported in Fig. 2(e). In fact, the net charges on $\mathrm{Pd}$ and $\mathrm{Ag}$ are found to be similar. In both cases, roughly 0.2 electrons are withdrawn from the metal atom per metal-oxygen bond. That opposite trends in CLS upon oxidation are obtained for $\mathrm{Pd}$ and $\mathrm{Ag}$ despite similar interatomic charge transfers is another example of the limitations of the electrostatic potential model to rationalize shifts in binding energies of core electrons.

To understand the different trend for $\mathrm{Pd}$ and $\mathrm{Ag}$ upon oxidation, we investigate the difference in bond characteristics by analyzing the projected density of states (PDOS). In Fig. 3, the metal $d$ component of a surface atom of a clean surface is compared to the case with 0.75 coverage of oxygen. The focus is put on $d$ states as these can be used to monitor differences in chemical properties of transition metals. ${ }^{28}$ To see which states should be assigned to the metal-oxygen bond, also the oxygen $p$ components are reported.

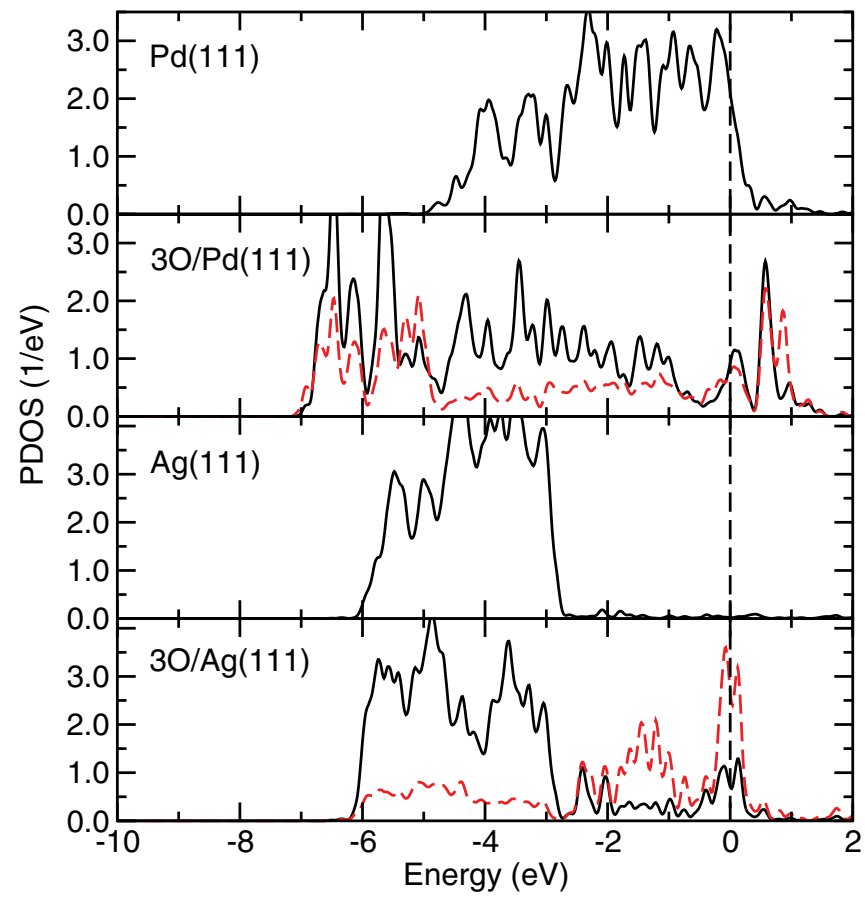

FIG. 3. (Color online) Projected electronic density of states from top to bottom of $\mathrm{Pd}(111), 3 \mathrm{O} / \mathrm{Pd}(111), \mathrm{Ag}(111)$, and 3O/ $\mathrm{Ag}(111)$. The energies are reported with respect to the corresponding Fermi energy. The solid (dashed) lines correspond to projection on metal $4 d$ (oxygen $2 p$ ). 
For Pd, the $d$ band crosses the Fermi energy and broadens upon oxidation. The width of the $d$ band for the clean surface is $\sim 5 \mathrm{eV}$, whereas it is $\sim 7 \mathrm{eV}$ for the oxidized case. The broadening is related to the formation of metal-oxygen bonds. Bonding combinations are found at low energies, whereas antibonding states are located mainly above the Fermi energy. The situation is noticeably different for Ag. The $d$ band is for the clean surface located $\sim 3 \mathrm{eV}$ below the Fermi energy, and oxidation affects the width of these states only in a minor fashion. In particular, the lower edge of the $d$-derived states is virtually not shifted at all with respect to the Fermi level. There are new features at higher energies that could be assigned to bonding and antibonding combinations of $\mathrm{Ag} 3 d$ and $\mathrm{O} 2 p$. However, in contrast to oxidized Pd, both sets of orbitals are located below the Fermi energy. From the comparison of the valence states of $\mathrm{Pd}$ and $\mathrm{Ag}$, it can be concluded that the bonding of oxygen to the two metals is different in character. For $\mathrm{Pd}$, the bonding includes contributions of the $d$ states, whereas for $\mathrm{Ag}$, the bonding should be attributed mainly to $s$ states.

In addition, to monitor the character of the bonding, the $d$ states in the valence could be used to investigate the change in the onsite electrostatic potential. ${ }^{12}$ In particular, the shift of the center of the $d$ band $\left(\Delta \varepsilon_{d}\right)$ with respect to the Fermi energy is a measure of the change in the electrostatic potential. From the PDOS analysis, it is obvious that the center of the $d$ band is shifted to lower energies for Pd, whereas it is virtually unchanged for Ag. The shifts of the center of the $d$ band are reported in Fig. 2(f). For Pd, there is a clear linear relation between the number of oxygen bonds (or CLS) and $\Delta \varepsilon_{d}$. The lower the $d$-band center, the more positive is the CLS. Interestingly, the $\Delta \varepsilon_{d}$ for $\mathrm{Ag}$ is close to unchanged upon oxidation. The calculated values are within $0.1 \mathrm{eV}$ and there is no correlation with the number of $\mathrm{O}-\mathrm{Ag}$ bonds.

The CLS without final-state effects can in the pseudopotential method be estimated by calculating the variation in total energy when the pseudopotential without a core hole $\left(V^{\mathrm{PP}}\right)$ is replaced by one with a core hole $\left(V_{\mathrm{CH}}^{\mathrm{PP}}\right) .{ }^{29}$ Thus, the expectation value of

$$
\Delta V=V^{\mathrm{PP}}-V_{\mathrm{CH}}^{\mathrm{PP}}
$$

with an electronic density that corresponds to the unperturbed ground state. The results of such calculations using the QUANTUM-ESPRESSO package ${ }^{30,31}$ are shown in Fig. 2(f). The trends for $\Delta V$ are similar to those of $\Delta \varepsilon_{d}$. There is a linear correlation between the number of metal-O bonds (or CLS) and $\Delta V$ for $\mathrm{Pd}$. For $\mathrm{Ag}$, on the other hand, the measure of the initial-state effects is not dependent on oxidation state.
The analysis of $\Delta \varepsilon_{d}$ and $\Delta V$ reveals that the CLS for Pd upon oxidation to a large extent can be related to initial-state effects. On the contrary, the negative shifts for Ag should be attributed to final-state effects. As already mentioned, the screening configuration of $\mathrm{Ag}$ is $d^{10} s^{2}$. As charge has been depleted from the $s$ band of $\mathrm{Ag}$ upon oxidation (the $d$ band is close to unchanged), the extra electron that screens the core hole occupies states with bonding character. This is in contrast to the bulk reference atom, where the screening electron occupies antibonding states.

The character of the state occupied by the screening electron on the site with a core hole can be probed by comparing the oxygen atomic binding energy with a hypothetical system where the atom with the core hole has been replaced by silver (in the case of $\mathrm{Pd}$ ) and cadmium (in the case of $\mathrm{Ag}$ ). ${ }^{32} \mathrm{Ag}$ (Cd) has the same electronic configuration as the screening configuration of $\mathrm{Pd}(\mathrm{Ag})$. This comparison was done at an oxygen coverage of 0.75 . The binding energy (per oxygen atom) is decreased by $0.6 \mathrm{eV}$ when $\mathrm{Pd}$ is replaced by $\mathrm{Ag}$. The situation is reversed for $\mathrm{Ag}$, where instead the oxygen binding energy is increased by $0.2 \mathrm{eV}$. Thus, the extra electron on the $\mathrm{Pd}$ atom with a core hole occupies antibonding states, whereas bonding states are occupied in the case of $\mathrm{Ag}$.

\section{CONCLUSIONS}

The density functional theory and high-resolution corelevel spectroscopy have been used to explore the CLS of $\mathrm{Ag} 3 d$ upon oxidation. In particular, a comparison with the corresponding $\mathrm{Pd} 3 d$ shifts has been performed. Pd shows normal positive shifts of the electronic binding energies, whereas the shifts for Ag are negative. Analysis of initial-state effects and valence electronic structure shows that the onsite Ag core potential is insensitive to oxidation despite a clear metal-to-oxygen charge transfer. This is in contrast to Pd, where the onsite potential is deeper in the case of an oxidized metal atom. Thus, whereas the shifts for Pd can be rationalized in an initial-state picture, the marked negative shifts for oxidized Ag should be attributed to final-state effects where screening of the core hole takes place in $s$-derived states of bonding character.

\section{ACKNOWLEDGMENTS}

H.G. thanks Weine Olovsson for valuable discussions. Support from the Swedish Foundation for Strategic Research and the Swedish Research Council is acknowledged. The calculations have been performed at C3SE (Göteborg) and PDC (Stockholm).

\footnotetext{
*ghj@chalmers.se

${ }^{1}$ K. Siegbahn, Rev. Mod. Phys. 54, 709 (1982).

${ }^{2}$ P. Kostelnik, N. Seriani, G. Kresse, A. Mikkelsen, E. Lundgren, V. Blum, T. Sikola, P. Varga, and M. Schmid, Surf. Sci. 601, 1574 (2007).

${ }^{3}$ J. Schnadt, A. Michaelides, J. Knudsen, R. T. Vang, K. Reuter, E. Lægsgaard, M. Scheffler, and F. Besenbacher, Phys. Rev. Lett. 96, 146101 (2006).
}

${ }^{4}$ M. Schmid, A. Reicho, A. Stierle, I. Costina, J. Klikovits, P. Kostelnik, O. Dubay, G. Kresse, J. Gustafson, E. Lundgren et al., Phys. Rev. Lett. 96, 146102 (2006).

${ }^{5}$ B. Lindberg, K. Hamrin, G. Johansson, U. Gelius, A. Fahlman, C. Nordling, and K. Siegbahn, Phys. Scr. 1, 286 (1970).

${ }^{6}$ H. Gelius, Phys. Scr. 9, 133 (1974).

${ }^{7}$ N. Mårtensson and A. Nilsson, J. Electron Spectrosc. Relat. Phenom. 75, 209 (1995). 
${ }^{8}$ L. H. Tjeng, M. B. J. Meinders, J. van Elp, J. Ghijsen, G. A. Sawatzky, and R. L. Johnson, Phys. Rev. B 41, 3190 (1990).

${ }^{9}$ V. K. Kaushik, J. Electron Spectrosc. Relat. Phenom. 56, 273 (1991).

${ }^{10}$ J. N. Andersen, D. Hennig, E. Lundgren, M. Methfessel, R. Nyholm, and M. Scheffler, Phys. Rev. B 50, 17525 (1994).

${ }^{11}$ M. Weinert and R. E. Watson, Phys. Rev. B 51, 17168 (1995).

${ }^{12}$ I. A. Abrikosov, W. Olovsson, and B. Johansson, Phys. Rev. Lett. 87, 176403 (2001).

${ }^{13}$ P. Steiner and S. Hufner, Acta Metall. 29, 1885 (1981).

${ }^{14}$ S. J. Clark, M. D. Segall, C. J. Pickard, P. J. Hasnip, M. J. Probert, K. Refson, and M. C. Payne, Z. Kristallogr. 220, 567 (2005).

${ }^{15}$ We use the CASTEP program.

${ }^{16}$ J. P. Perdew, K. Burke, and M. Ernzerhof, Phys. Rev. Lett. 77, 3865 (1996).

${ }^{17}$ D. Vanderbilt, Phys. Rev. B 41, 7892 (1990).

${ }^{18}$ E. Pehlke and M. Scheffler, Phys. Rev. Lett. 71, 2338 (1993).

${ }^{19}$ R. Nyholm, J. N. Anderssen, U. Johansson, B. Jensen, and I. Lindau, Nucl. Instrum. Methods Phys. Res., Sect. A 467, 520 (2001).

${ }^{20}$ M. Todorova, E. Lundgren, V. Blum, A. Mikkelsen, S. Gray, J. Gustafson, M. Borg, J. Rogal, K. Reuter, J. N. Andersen et al., Surf. Sci. 541, 101 (2003).

${ }^{21}$ R. Westerström, M. E. Messing, S. Blomberg, A. Hellman, H. Grönbeck, J. Gustafson, N. M. Martin, O. Balmes, R. van Rijn, J. N. Andersen et al., Phys. Rev. B 83, 115440 (2011).

${ }^{22}$ The calculated shifts for the five unique atoms are $-0.24,-0.23$, $-0.14,0.20$, and $0.26 \mathrm{eV}$, respectively.
${ }^{23}$ This has been verified by explicit calculations for the structurally resolved $c(4 \times 8)$ overlayer reported in Ref. 33 . The stoichiometry of the oxide layer in $c(4 \times 8)$ is $\mathrm{Ag}_{5} \mathrm{O}_{4}$ and we calculate CLS of $-0.54 \mathrm{eV}$ and $-1.05 \mathrm{eV}$ with respect to the bulk component.

${ }^{24}$ B. Johansson and N. Mårtensson, Phys. Rev. B 21, 4427 (1980).

${ }^{25}$ A. Rosengren and B. Johansson, Phys. Rev. B 23, 3852 (1981).

${ }^{26} \mathrm{R}$. Bader, Atoms in Molecules: A Quantum Theory (Oxford University Press, New York, 1990).

${ }^{27}$ G. Henkelman, A. Arnaldsson, and H. Jónsson, Comput. Mater. Sci. 36, 354 (2006).

${ }^{28}$ B. Hammer and J.K. Nørskov, Adv. Catal. 45, 71 (2000).

${ }^{29}$ L. Bianchettin, A. Baraldi, S. de Gironcoli, S. Lizzit, L. Petaccia, E. Vesselli, G. Comelli, and R. Rosei, Phys. Rev. B 74, 045430 (2006).

${ }^{30}$ S. Baroni, A. Dal Corso, S. de Gironcoli, P. Giannozzi, C. Cavazzoni, G. Ballabio, S. Scandolo, G. Chiarotti, P. Focher, A. Pasquarello, K. Laasonen, A. Trave, R. Car, N. Marzari, and A. Kokalj, http://www.pwscf.org/.

31 Norm-conserving pseudopotentials (Ref. 34) are used and the Kohn-Sham orbitals are expanded with a 62-Ry plane-wave kinetic energy cutoff.

${ }^{32}$ Changes in adsorbate binding energies are in the, so-called, $Z+1$ approach (Ref. 7) related to changes in the core-level shifts. In fact, the change in adsorbate binding energy equals the CLS provided that a $Z+1$ impurity in the bulk of the metal does not affect the adsorbate binding energy.

${ }^{33}$ J. Schnadt, J. Knudsen, X. L. Hu, A. Michaelides, R. T. Vang, K. Reuter, Z. Li, E. Lægsgaard, M. Scheffler, and F. Besenbacher, Phys. Rev. B 80, 075424 (2009).

${ }^{34}$ N. Troullier and J. L. Martins, Phys. Rev. B 43, 1993 (1991). 